\title{
EL NIVEL DE VIDA
}

\author{
¿Hay alguna ley de la Naturaleza o, más \\ bien, \\ alguna ley de Dios por la cual un hombre tiene el \\ derecho a disponer de comida suficiente, ropa su- \\ ficiente, abrigo suficiente y recreación suficiente, \\ sólo porque trabaja?
}

Anthony Trollope

\section{Introducción}

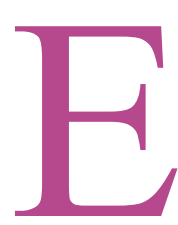

xplícita o implícitamente la filosofía política se ha ocupado del tema del nivel de vida. El tratamiento específico más completo de la cuestión lo constituye el reciente trabajo de Amartya Sen The Standard of Living ${ }^{1}$. Sin embargo, me parece que ese trabajo arriba a una solución insatisfactoria, por lo que vale la pena compararlo con una idea más simple que voy a sostener aquí. Parcialmente expuesta, la idea es que el nivel de vida depende de la opulencia, entendida de este modo: el nivel de vida está determinado por la cantidad de bienes que una persona tiene a su disposición. Por bienes se entienden el ingreso y la riqueza; para simplificar propongo emplear como denominación única la palabra «opulencia»². Entonces, el nivel de vida se identifica con el nivel de opulencia.

Ante todo quiero aclarar que ésta no es la idea completa que voy a desarrollar; el tema es más complejo pero me parece adecuado complicarlo de a poco. Comenzaré entonces con la idea

${ }^{1}$ Amartya Sen, The Standard of Living, Cambridge University Press, 1987.

${ }^{2}$ Claro que la simplificación puede ser excesiva porque el problema no es tan sencillo como parece. Supongamos que respecto de todos los bienes que están en consideración el individuo $x$ tiene más cantidad de ellos que el individuo $y$. En ese caso resulta claro que $x$ tiene más riqueza que $y$. Pero no ocurre lo mismo si $x$ tiene más cantidad de algunos bienes e $y$ de otros. ¿Quién tiene en este caso más riqueza? Aunque la solución no está exenta de toda arbitrariedad, propongo comparar los paquetes de bienes de $x$ e $y$ respecto de un patrón común de medida, la moneda. El paquete de bienes que se cotiza más alto indica que su propietario tiene más riqueza. 
de opulencia y agregaré luego otros elementos, tales como el tiempo libre, la libertad de elección y la calidad de vida. Pasaré luego del caso de una sola persona al de varias. Una vez completado el modelo lo compararé con el propuesto por Sen y con el modelo basado en la satisfacción de las necesidades. Concluiré mostrando un problema específico que afecta a la libertad de elección.

Aunque supongo que resulta obvio, quiero aclarar que me ocuparé del nivel de vida tal como puede obtenerse sin violar las normas jurídicas de la sociedad que se toma como referencia. No me ocuparé por ejemplo, de:

El caso del farsante exitoso: $x$ carece de bienes pero tiene dotes persuasivas que le permiten obtener préstamos importantes de dinero, los que no restituye. Pese a tener solamente deudas, vive cómodamente y con tiempo libre a su disposición.

Pero $x$ puede encontrarse con un acreedor insatisfecho que le recuerde súbitamente que las deudas deben pagarse, de acuerdo al sistema jurídico en el que ambos viven, y lo obligue a hacerlo.

\section{El modelo más simple}

Tomemos para comenzar el caso de una sola persona. Si el único elemento a tomar en cuenta para determinar su nivel de vida es su grado de opulencia, entonces cuanto más opulenta sea una persona mayor nivel de vida tendrá como consecuencia. La figura 1 describe esta circunstancia.

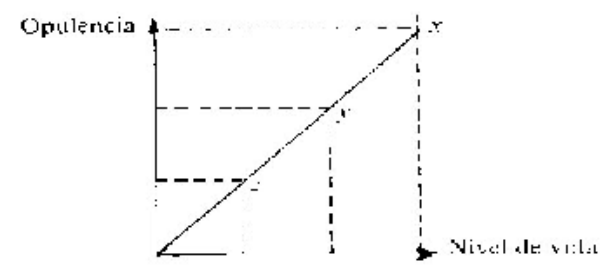

Figura 1

Lo que la figura 1 se limita a mostrar es que cada unidad de opulencia equivale a una unidad en el nivel de vida. Puesto que $z$ 
tiene una unidad de opulencia tiene también una unidad en el nivel de vida; y tiene dos de ambos y $x$ tiene tres unidades.

La opulencia se refleja en la columna vertical y el nivel de vida en la columna horizontal. La opulencia aumenta a medida que nos desplazamos hacia arriba y el nivel de vida aumenta a medida que nos desplazamos a la derecha. (El lector debe abstenerse de extraer conclusiones políticas de esta última afirmación.) Lo que la figura muestra es que el grado de opulencia, como he dicho, se refleja directamente en el nivel de vida. Como $x$ es más opulento que $z$ tiene un mejor nivel de vida que éste, por ejemplo ${ }^{3}$.

Al identificar en esta primera aproximación al nivel de vida con la opulencia no estoy sosteniendo que la opulencia sea el dato más relevante para evaluar moralmente al sistema; es sólo el dato más relevante para evaluar el nivel de vida que se disfruta dentro del sistema. Luego veremos si lo único que caracteriza a la moralidad del sistema es su éxito en incrementar la opulencia (y la conclusión será negativa). Pero no hay duda que el nivel de vida importa para determinar lo que un gobierno debe hacer. Pocos estarían dispuestos a discutir la idea de que el gobierno debe intentar elevar el nivel de vida de sus súbditos. Y esto muestra una diferencia entre dos conceptos -nivel de vida y bienestar- cuya importancia se hará explícita más adelante. Un gobierno puede tener éxito en su propósito de elevar el nivel de vida y -aún así- sus súbditos pueden no ser felices, en el sentido de no disfrutar de bienestar ${ }^{4}$.

${ }^{3}$ Uno de los defectos del gráfico propuesto es que parece mostrar demasiado. Transmite la impresión, por ejemplo, de que $x, y$ y $z$ : tienen el mismo número de unidades de opulencia y de nivel de vida. Sin embargo, yo sólo pretendo introducir al nivel de vida como una nación ordinal y no cardinal (entre otras cosas, porque intentar lo contrario es mucho más complicado).

${ }^{4}$ Pero aquí hay que distinguir dos casos diferentes: a) los súbditos no disfrutan de bienestar pese a tener un nivel de vida alto, $\mathrm{y}$ b) los súbditos no disfrutan de bienestar porque tienen un nivel de vida alto. El caso a) es sencillo: el gobierno debe seguir tratando de elevar el nivel de vida, porque no es el nivel de vida alto el que incide en la falta de bienestar (al contrario, bien podemos imaginar que la atenúa). El caso b) es más complicado. Creo que debemos dividirlo en dos subcasos: $\mathrm{b}_{1}$ ) El individuo $x$ no disfruta de bienestar porque él tiene un nivel de vida alto. Este subcaso vuelve a ser sencillo de solucionar. El gobierno debe seguir tratando de elevar el nivel de vida de los súbditos, y $x$ puede aumentar su bienestar desprendiéndose de sus bienes y disminuyendo su nivel de vida. $\mathrm{b}_{2}$ ) El individuo y no disfruta de bienestar porque él y los demás tienen un nivel de vida alto. Ahora tampoco el problema parece tan complicado. El gobierno sigue teniendo la obligación de elevar el nivel de vida. Las preferencias externas de $y$ no tienen por qué tener prioridad 


\section{El modelo se complica}

Hasta ahora el problema parece no ser muy complejo (ni tampoco muy interesante): a mayor opulencia, mayor nivel de vida. Supongamos que $x$ e $y$ tengan exactamente el mismo nivel de opulencia. Sin embargo, a $x$ le alcanza con cuatro horas diarias de trabajo para lograr ese grado, mientras que $y$ necesita diez horas. ¿Puede decirse que ambos tienen el mismo nivel de vida? Parece que la respuesta es negativa, lo que muestra que el modelo inicial es excesivamente simple. El tiempo libre tiene un valor en el cómputo del nivel de vida. Si consideramos al tiempo libre como un «bien» $x$ tiene más bienes que $y$, porque $y$ tuvo que «vender» una parte mayor de su tiempo libre para «comprar» su grado de opulencia. Esto se muestra en la figura 2.

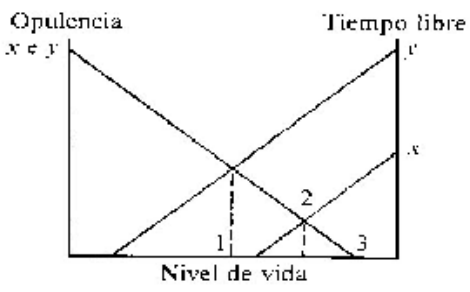

Figura 2

La columna vertical de la derecha incorpora al cálculo el nuevo dato del tiempo libre. El tiempo libre aumenta a medida en que nos desplazamos hacia abajo en esa columna. Ateniéndonos sólo a la opulencia $x$ e $y$ tienen el mismo nivel de vida, esto es, el punto 3 . Agregando el elemento del tiempo libre $x$ tiene un mejor nivel de vida que $y$ : el nivel de vida de $x$ se encuentra en el punto 2 y el de y en el punto 1. Por razones de simplicidad la figura asigna igual valor a la opulencia y al tiempo libre. Obviamente los valores varían de individuo a individuo, de acuerdo a las preferencias personales (y a otros factores sobre los que volveré luego) 5. En la figura el nivel de vida se

sobre las preferencias personales de los demás súbditos. Cfr. Ronald Dworkin, Taking Rights Seriously, London, Duckworth, 1977, págs. 235 y sigs.

${ }^{5}$ Esto no significa que el criterio no sea objetivo. Como luego se verá, combina elementos objetivos y subjetivos, estos últimos vinculados con la satisfacción de las preferencias. 
determina por la intersección de las líneas correspondientes a ambos elementos.

Pero la figura 2 sólo tiene el mérito de la simplicidad y no debe ser considerada como una representación adecuada del nivel de vida. Porque, entre otras cosas, es posible que las líneas de $x$ e $y$ no se intersecten, o que no pueda trazarse la línea de $x$ para determinar su nivel de vida porque tiene la máxima cantidad de tiempo libre y el mínimo nivel de opulencia (es el caso del desempleado, por ejemplo, del que me ocuparé luego). De modo que la figura 2 no tiene otro propósito que el de llamar la atención sobre una circunstancia: que dos personas con idéntico grado de opulencia pueden diferir en nivel de vida.

Lo que la figura 2 muestra, entonces, es que el nivel de vida se incrementa al aumentar el tiempo libre ${ }^{6}$. Por tanto, el gobierno puede elevar el nivel de vida de los súbditos no sólo propiciando un aumento de salarios, sino también consiguiendo un número mayor de días de vacaciones pagadas.

\section{Aparece un elemento adicional}

El modelo ya es ahora algo más complejo. Pero todavía no refleja todos los elementos de la realidad que podrían contar para el cálculo del nivel de vida. Supongamos que $x$ e $y$ viven en dos sociedades distintas, Libertas y Servitudo. Ambos tienen el mismo grado de opulencia y de tiempo libre. Pero difieren en algo muy importante: en Libertas $x$ tiene un alto grado de libertad para decidir qué hacer con sus bienes y con su tiempo libre, mientras que en Servitudo el gobierno limita severamente las alternativas de $y$. (El caso límite es el de $z$, que vive en Totalitarias: el gobierno le indica exactamente cómo debe gastar su dinero y emplear el tiempo que no dedica a trabajar) ¿ ¿Diríamos que $x$ e $y$ tienen el mismo nivel de vida? La figura 2 nos dice que debemos responder que sí, pero tal vez esto indica que algo anda mal en la figura.

${ }^{6}$ Conviene precisar algo más la idea de «tiempo libre». Yo la entiendo como el tiempo disponible una vez cumplido con las actividades necesarias para alcanzar el grado de opulencia de que se disfruta, y con el dedicado a descansar y alimentarse. Si yo me comprometo a ir al cine con un amigo, en cierto sentido ese compromiso indica que ese tiempo ya no es «libre». Pero lo es en la acepción que estoy empleando aquí, porque no lo dedico ni a descansar ni a incrementar mi nivel de opulencia.

${ }^{7}$ Nótese que digo «tiempo que no dedica a trabajar». Aquí no tendría sentido hablar de tiempo libre. 
Veamos el asunto más de cerca. La opulencia y el tiempo libre constituyen condiciones necesarias para disfrutar de libertad positiva, entendida como «libertad para», como posibilidades de llevar a cabo ciertos planes de vida. (La opulencia y el tiempo libre pueden incluso identificarse con la libertad positiva) ${ }^{8}$. Pero en el caso de un solo individuo la libertad positiva misma requiere de una condición necesaria: la libertad negativa, la «libertad de», entendida como ausencia de restricciones jurídicas ${ }^{9}$. En Libertas $x$ tiene ambas libertades; disfruta de un cierto grado de opulencia y de tiempo libre y no tiene restricciones para decidir qué hacer con ellos. En Servitudo $y$ no tiene libertad negativa debido a las restricciones del gobierno. Y puesto que la libertad negativa es condición necesaria de la libertad positiva ${ }^{10}$, tampoco disfruta de esta última ${ }^{11}$. (Me limito a estos ejemplos por razones de simplicidad, pero no es difícil imaginar otros. En Proletarias los súbditos pueden gozar de una gran libertad negativa, pero ser tan pobres que nunca pueden convertirla en libertad positiva.)

¿Cómo influye todo esto en la determinación del nivel de vida? Acá se presentan dos alternativas posibles. La primera es considerar que la libertad cuenta para la determinación del nivel de vida y esto, a su vez, puede interpretarse de dos maneras diferentes: a) que la libertad es un bien del mismo tipo que el ingreso y la riqueza, por lo que su cantidad cuenta en el cálculo del grado de opulencia, o b) que la libertad es un bien de distinto tipo, que debe calcularse independientemente de la opulencia, tal como ocurre con el tiempo libre. La otra alternativa es entender que la libertad no cuenta para la determinación del nivel de vida, pero sí para establecer la deseabilidad del sistema: ante dos sistemas que otorgan el mismo nivel de vida uno puede ser más

${ }^{8}$ Esta afirmación no queda desmentida por la circunstancia de que en algunos planes de vida no figuren la opulencia y el tiempo libre. San Francisco de Asís, por ejemplo, no deseaba disponer de tiempo libre, pero podríamos decir que utilizaba su tiempo para predicar contra la pérdida de tiempo. Tampoco era partidario de la riqueza, pero como sí lo era de la caridad, destinaba su opulencia a ese fin.

${ }^{9} \mathrm{Me}$ concentro en las restricciones jurídicas porque estoy evaluando los sistemas jurídicos vigentes en Libertas y Servitudo. Las restricciones ilegítimas, sin embargo, también cuentan para evaluar el grado de libertad negativa.

${ }^{10}$ Por lo menos si se quiere ejercer la libertad positiva de un modo legal, no ilegal. En Argentina suele existir la libertad positiva de comprar dólares, aunque no la libertad negativa de hacerlo.

${ }^{11}$ La relación entre la libertad negativa y la positiva se examina en el capítulo dedicado a ese tema. No me extiendo por ello aquí en detalle. 
deseable que otro por conceder un mayor grado de libertad de elección. El cuadro 1 refleja estas alternativas.

\section{Cuadro 1}

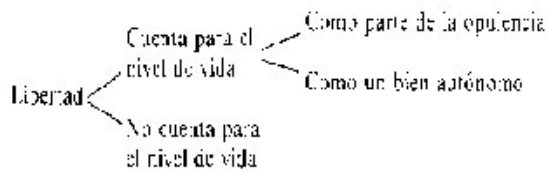

La primera alternativa me parece muy complicada para adoptar. La libertad está conectada con la opulencia de diversas maneras, como hemos visto. La opulencia se identifica con la libertad positiva y la libertad negativa es condición necesaria de la positiva en el caso de un solo individuo. Considerar a la libertad como un bien del mismo tipo que el ingreso y la riqueza es sólo introducir un elemento de confusión en el cálculo.

La segunda alternativa no es muy diferente de la tercera, contra lo que podría parecer. Por una parte, computa la opulencia y el tiempo libre, y, por la otra, el grado de libertad para elegir qué hacer con ellos. La diferencia reside en esto: si el grado de libertad de elección es bajo, la segunda alternativa considera que baja el nivel de vida. Supongamos que $x$ tenga un alto grado de opulencia y de tiempo libre. Como alternativas viables de elección tiene las posibilidades A, B, C, D y E. (Las tiene tanto como conjunciones cuanto como disyunciones, inclusivas o exclusivas.) El gobierno, sin embargo, le prohíbe optar por D y por E. Pero de hecho $x$ sólo pensaba elegir A o B (o ambas, a lo mejor). Parecería curioso decir que en este caso el nivel de vida de $x$ ha disminuido como consecuencia de la acción del gobierno.

No obstante, sin duda es preferible poder elegir entre más opciones, aunque sea para descartarlas. El interés de $x$ por mantener abiertas las opciones D y E puede ser de tipo prudencial: él no descarta la posibilidad de que puedan interesarle en el futuro (o la posibilidad de que le interesen a sus descendientes). Aunque el cierre de esas opciones no afecta su nivel de vida, él preferiría un sistema que las permitiera. (El $x$ que estoy describiendo es -como se ve- un ciudadano liberal; un ciudadano autoritario estaría encantado de que se prohibieran las opciones que no le gustan.) Distingue, entonces, entre el nivel de vida y la deseabilidad del sistema. Esta es la alternativa que me parece más 
aceptable. El nivel de vida depende sólo de la opulencia y del tiempo libre. Pero de dos sistemas con idéntico nivel de vida uno puede ser más deseable que otro, dependiendo la deseabilidad del grado de libertad de elección (y de otro factor que introduciré a continuación).

\section{El problema de los bienes públicos}

El modelo original es ahora algo más complicado. El nivel de vida de una persona depende de una combinación de dos factores, su opulencia y su tiempo libre. Y dos sistemas con igual nivel de vida pueden tener distintos grados de deseabilidad de acuerdo a sus respectivos grados de libertad de elección. Ésta no es todavía la pintura completa. Supongamos que $x$ e $y$ viven en dos sociedades diferentes, que poseen al mismo nivel de vida (de acuerdo a los parámetros vistos hasta ahora) y el mismo grado de libertad de elección; llamémoslas Ecológicas y Polutas.

Ecológicas disfruta de aire puro; sin embargo, mientras Polutas sufre los efectos de la contaminación. ¿Podemos decir que el nivel de vida de ambas es el mismo? Si no lo es, los parámetros que hemos considerado hasta ahora son insuficientes. Como es obvio, el caso que he presentado es el de los bienes públicos. Un bien público presenta dos características: $\left.1 .^{\circ}\right)$ el consumo de una persona respecto de ese bien no reduce la capacidad de otras personas para disfrutarlo, y $2^{\circ} .^{\circ}$ una vez que el bien es producido no hay manera eficiente de excluir a alguien de su consumo ${ }^{12}$. El aire puro es un ejemplo típico de bien público y la defensa nacional es otro.

Si un tercer individuo -z- tuviera que elegir entre vivir en Ecológicas o vivir en Polutas parece plausible pensar que elegiría la primer alternativa. Esto no pienso discutirlo, por supuesto. Lo que me interesa establecer es si $z$ elegiría Ecológicas por considerar que tiene un mejor nivel de vida. Un aspecto interesante del tema es que el caso de los bienes públicos sólo puede tener importancia (si es que la tiene) para calcular el nivel de vida en el caso de que comparemos sistemas distintos, esto es, en una comparación intersistemática. Dentro de un mismo sistema los bienes públicos cuentan por igual en el nivel de vida de todos los habitantes. En el caso en que no lo hacen el factor que desequilibra

\footnotetext{
${ }^{12}$ Jules L. Coleman, Markets, Morals and the Law, Cambridge University Press, 1988, pág.
} 253. 
es la opulencia: $x$ no resulta afectado por la falta de parques públicos porque puede afrontar la compra de una casa con jardín privado, mientras que $y$-que no puede sufragarla- sí resulta afectado por la falta de parques. Pero la opulencia es el principal factor que ya he computado $^{13}$.

Me parece que con los bienes públicos (como el aire puro) ocurre algo similar que con la libertad de elección: no integran el nivel de vida pero juegan un papel en el momento de optar, como ocurre cuando $z$ debe elegir. El aire puro no integra el nivel de vida, sino la calidad de vida. El nivel de vida de Tokyo es medianamente alto, medido por la combinación de dos factores: a) el factor opulencia, que es muy alto, y b) el factor tiempo libre, que es relativamente bajo. La libertad de elección del sistema es alta, puesto que el gobierno no limita las opciones de los ciudadanos. Pero la calidad de vida es baja, pues los parques públicos no abundan y la contaminación del aire es elevada.

Faltaba entonces un factor a considerar cuando examinamos la deseabilidad del sistema: el grado en que se provean ciertos bienes públicos, la calidad de vida. El panorama completo en el caso de un individuo es entonces éste: su nivel de vida depende del grado de opulencia y del tiempo libre. La deseabilidad del sistema en que vive depende del grado de libertad de elección y de la calidad de vida. Por eso no es contradictoria, ni perplejante, la conducta de un individuo que emigra de un país a otro con igual nivel de vida; porque el segundo sistema puede ser más deseable que el primero.

Una aclaración se impone aquí: al limitar la deseabilidad del sistema a la libertad de elección y a la calidad de vida estoy suponiendo que si comparamos entre dos sistemas sus niveles de vida son iguales. Si los niveles son distintos no es contradictorio -obviamenteconsiderar más deseable al sistema con un mejor nivel de vida, aunque sus otros factores de deseabilidad sean menores. (El aire es más puro en la Costa de Marfil que en Tokyo, por ejemplo.)

\section{De una persona a muchas}

Hasta este momento me he estado ocupando del nivel de vida desde el punto de vista de una sola persona. La cuestión se

${ }^{13}$ En el apartado 7 muestro por qué considero que la opulencia puede ser considerada como factor principal. 
complica, por supuesto, si se quiere examinar el nivel de vida de una sociedad. ¿Cómo medir en este caso la opulencia y el tiempo libre? Para simplificar el problema concentrémonos por el momento en la medición de la opulencia.

Desde luego que una sociedad no es nada más que un conjunto de individuos. (El «desde luego» irritaría a los hegelianos, pero es mi punto de vista y no voy a defenderlo aquí. Acepto -sí- que el concepto de sociedad incluye no sólo los individuos, sino las relaciones entre ellos, pero no voy más lejos.) Entonces, una manera de calcular la opulencia de una sociedad es sumando la opulencia de todos sus miembros. Sin embargo, salvo el caso poco probable de una distribución igualitaria de bienes, esa suma no reflejaría el nivel de vida de la sociedad. Supongamos que la sociedad M está integrada por tres individuos, $x$, $y$ y $z$. La opulencia de $x$ se calcula en 10 unidades, la de $y$ en 190 y la de $z$ en 1.000 unidades. Decir que la opulencia de la sociedad M equivale a 1.200 unidades no es decir nada desde el punto de vista del nivel de vida. Dividir las 1.200 unidades por el número de integrantes de M y afirmar que en M la opulencia promedio es de 400 unidades es distorsionar grotescamente la situación: ninguno de sus integrantes se acerca a esa suma. Tampoco podemos calcular la opulencia sobre la base del «hombre representativo», porque ni $x$, ni $y$ ni $z$ tienen algún título especial para merecer ese rol.

Si la distribución de la opulencia no es igualitaria lo que interesa es el grado de opulencia del grupo mayoritario. En sociedades reales, integradas por mucho más de tres personas, pueden detectarse distintos grupos sociales con diferentes grados de opulencia. $\mathrm{Si}$ uno de ellos es notoriamente mayoritario ${ }^{14}$, constituye un buen candidato para que su grado de opulencia se utilice para calcular el nivel de vida de la sociedad.

De no existir un grupo definidamente mayoritario es un error hablar en ese caso del nivel de vida de la sociedad M, como un todo. Hay que hablar, en cambio, de los diferentes niveles de vida de los distintos grupos sociales de $\mathrm{M}$. El tema se vuelve todavía un poco más complejo cuando la opulencia debe complementarse con el cálculo del tiempo libre. No siempre ambos factores varían concomitantemente. Los filósofos tienen más tiempo libre pero menos opulencia que los ejecutivos, por ejemplo.

${ }^{14} \mathrm{La}$ expresión «notoriamente mayoritario» es vaga. Pero esa vaguedad puede reducirse, estableciendo -por ejemplo- un determinado porcentaje para autorizar el uso de la expresión. No es necesario decidir aquí cuál debe ser ese porcentaje. 
Pero las reglas del cálculo no varían. Si hay un grupo claramente mayoritario se identifican esos dos factores (opulencia y tiempo libre) y se puede extender el resultado a todo el grupo social. Es razonable decir que el nivel de vida de la India es bajo aunque algunos rajás posean fortunas enormes. (Nuevamente en este caso, pretender que se promedien los ingresos de los rajás y de los intocables, por ejemplo, distorsionaría gravemente la situación.) En caso de no haber grupos notoriamente mayoritarios se analizan la opulencia y el tiempo libre de cada uno de los grupos. En ese caso no podrá hablarse del nivel de vida de la sociedad, sino de los niveles de vida de ella ${ }^{15}$.

Por supuesto que también en el caso de la sociedad el nivel de vida debe separarse de la deseabilidad del sistema, configurada por el grado de libertad de elección y por la calidad de vida.

Como se ve la tarea del gobierno en los casos de distribución no igualitaria es complicada. ¿Debe intentarse elevar el nivel de vida del grupo mayoritario? ¿O el del grupo peor situado» -O el de todos los grupos? Los utilitaristas darían una respuesta, Rawls otra, Pareto una tercera (la que podría considerarse una instancia de la respuesta utilitarista), y hay decenas más que están disponibles... Pero el tema de la justicia en relación con el nivel de vida no puede ser perseguido aquí. Hay otro elemento que tampoco tomo en consideración, y es en el de cómo adquirieron los respectivos grupos sociales -o individuos... su grado de opulencia y de tiempo libre. Mi análisis no se parece a una proyección cinematográfica, sino a una fotografía del instante presente.

\section{Complicaciones finales}

Todavía no ha concluido la tarea de complicar el modelo de modo que éste refleje las complicaciones de la realidad. Supongamos:

El caso del pobre desempleado: $x$ carece de empleo y no puede conseguirlo pese a sus esfuerzos. Su provisión de tiempo libre es enorme, puesto que comprende todo el día, salvo la pequeña parte de él que dedica a buscar trabajo. Su nivel de opulencia es nulo. pues durante $s u$ período de desempleo se han consumido todos sus ahorros.

\footnotetext{
${ }^{15}$ Obviamente, hay criterios estadísticos de medición que son más refinados.
} 
¿Quién querría computar todo el tiempo libre que $x$ tiene a su disposición para sostener que su nivel de vida no es bajo? Imaginemos que y tiene algunos bienes y cierto tiempo libre. ¿Diríamos que tiene el mismo nivel de vida que $x$, porque tiene más opulencia pero menos tiempo libre? La importancia del tiempo libre decrece ante la ausencia de opulencia (enseguida veremos que no ocurre exactamente lo mismo a la inversa). Tal vez estemos en presencia de la utilidad marginal decreciente, pero entonces nos enfrentamos con un problema: el cálculo de la utilidad marginal decreciente es subjetivo -por lo general- y aquí necesitamos un cálculo objetivo. Es posible conseguir un cálculo objetivo si asignamos un multiplicador a la opulencia y al tiempo libre, que aumenta si disminuye el objeto multiplicado. Por ejemplo: si la opulencia es escasa, se multiplica su valor por un número mayor para determinar su importancia para el individuo, y esta operación se realiza con independencia de las preferencias individuales. Un bien en exceso cuenta menos para el individuo, en este caso, y se supone que también debe contar menos para determinar su nivel de vida ${ }^{16}$.

Como anticipé, la situación de opulencia sin tiempo libre parece menos dramática para el cálculo del nivel de vida. Veamos:

El caso del empresario atareado: $z$ es un empresario de éxito, pero el éxito de su empresa requiere de su atención personal, sin que pueda delegar trabajo. La empresa ha crecido y la opulencia de $z$ condignamente ha aumentado. Pero debe dedicar todo su tiempo a la empresa, esto es, no tiene tiempo libre.

En este caso tal vez igual aceptaríamos decir que $z$ tiene un buen nivel de vida, aunque sin duda no tan alto como el de $y$, que obtiene los mismos ingresos de $z$ sin trabajar. Pero si aceptamos que $z$-a diferencia del desempleado- tiene un buen nivel de vida, lo que estamos diciendo es que la opulencia tiene más importancia que el tiempo libre para la determinación del nivel de vida. Mientras $x$, desempleado, sin bienes, y con todo su tiempo libre, no puede tener un buen nivel de vida, $z$, rico en bienes pero abrumado de trabajo, puede tenerlo. Creo que esto es así porque usualmente se supone que la opulencia es condición suficiente para conseguir tiempo libre, pero que la inversa es falsa.

${ }^{16}$ No pretendo ocultar que la elección del multiplicador puede contener un cierto grado de arbitrariedad, y que según cuál sea el multiplicador que se elija $x$ puede tener mayor nivel de vida que $y$, o resultar a la inversa. 
Nótese la implicancia de estas complicaciones finales. Primero, el valor de la opulencia y del tiempo libre depende del grado en que sean poseídos: a mayor grado, menor valor. Segundo, el valor de la opulencia es mayor que el del tiempo libre para determinar el nivel de vida. Lo que he expuesto son sólo sugerencias para un cálculo rudimentario del nivel de vida, cálculo mucho más complicado, como se ve, de lo que sugería la figura 2.

Pero todavía nos aguardan algunas complicaciones. ¿Qué ocurre con el individuo que trabaja intensamente, pero por placer, no por necesidad? Es:

El caso del director de orquesta: $w$, es un director de orquesta de gran éxito y prestigio. El disfruta enormemente con su trabajo. Sus honorarios son tan elevados que le bastaría con dirigir unos pocos conciertos por año, quedándole mucho tiempo libre a su disposición. Pero vi, sólo disfruta dirigiendo conciertos, y lo hace hasta quedar sin tiempo libre.

¿Hay que computar en este caso la falta de tiempo libre como un dato negativo para el nivel de vida de $w$ ? Ciertamente no. Él ha elegido voluntariamente -y por placer- no tener tiempo libre. En realidad, y en una acepción no desdefiable, él tiene tiempo libre. Porque «tiempo libre» es tiempo dedicado a lo que a uno le gusta, y a $w$ le gusta dirigir ${ }^{17}$. Sólo cuenta la falta de tiempo libre cuando es obligada, cuando el individuo debe reducir su tiempo libre para obtener el nivel de opulencia que requiere.

Pero el director de orquesta se encuentra en una situación distinta, en un sentido, a la que refleja:

El caso del trabajador afortunado: $y$ necesita trabajar intensamente para obtener el nivel de opulencia que requiere, pero su trabajo le agrada con igual intensidad. No tiene tiempo libre, ni puede tenerlo. Pero si pudiera elegir qué hacer con su tiempo libre, $y$ elegiría trabajar como lo hace.

En este ejemplo, puesto que $y$ no puede decidir el monto de su tiempo libre (a diferencia de lo que acontecía con $w$ ) yo creo que la falta de tiempo libre se computa para determinar su nivel de vida. Su buena fortuna, el agrado que experimenta en su trabajo, cuenta para determinar su bienestar, no su nivel de vida.

${ }^{17}$ Nótese que esto no convierte al tiempo libre en una noción subjetiva. En este caso $w$, tiene tiempo libre porque no dirige para aumentar su grado de opulencia, puesto que ya alcanzó el grado deseado. 


\section{Cómo evaluar el modelo}

El modelo propuesto tiene la ventaja de su sencillez. Calcula el nivel de vida únicamente sobre la base de dos elementos: la opulencia y el tiempo libre. Deja aparte a la libertad de elección y a la calidad de vida, considerándolos como parte de la deseabilidad del sistema. Si hay un grupo netamente mayoritario identifica al nivel de vida de ese grupo con el nivel de vida de la sociedad, y si no lo hay considera separadamente los niveles de vida de los distintos grupos sociales. Es cierto que el tema se complica cuando consideramos los diferentes valores que pueden asumir la opulencia y el tiempo libre, pero aún así se trata de un modelo tolerablemente simple. No obstante, la simplicidad no es garantía de corrección.

Otra ventaja aparente del modelo propuesto es que parece coincidir con el sentido que el lenguaje corriente asigna a la expresión «nivel de vida». Cuando alguien se refiere coloquialmente al nivel de vida de un individuo o de un grupo social está pensando en su opulencia y en su tiempo libre. Pero el modelo quiere ser algo más que la reconstrucción del modo habitual de expresarse. A fin de cuentas es posible que el hablante medio no aprecie las complejidades técnicas de los términos que emplea (esto ocurre con la palabra «democracia», por ejemplo).

De modo que el hecho de que el modelo sea simple y coloquial no cuenta decisivamente en su favor. Para evaluarlo existe un método mejor: cotejarlo con el modelo alternativo más conocido; si resulta más atrayente que éste las razones para sostenerlo se habrán robustecido. El modelo alternativo al que me refiero es -como dije al comienzo- el que diseñó Amartya Sen-.

\section{El modelo de Sen}

El estudio del modelo de Sen puede comenzar fructíferamente con el análisis de sus motivos para rechazar a la utilidad como criterio de evaluación del nivel de vida. Sen distingue tres maneras diferentes de concebir a la utilidad: $a$ ) como placer y felicidad; $b$ ) como la satisfacción de los deseos, y c) como elección.

a) En el caso del placer Sen observa ${ }^{18}$ que una persona pobre, explotada y enferma puede estar, sin embargo, satisfecha con su destino, tal vez debido al condicionamiento social. Puesto

\footnotetext{
${ }^{18}$ Sen, cit., pág. 8 .
} 
que el nivel de vida no puede ser separado del tipo de vida que la persona lleva, se requiere algún logro objetivo como parte del ser «realmente feliz».

b) En el caso de la satisfacción de los deseos Sen señala que la ausencia de deseos puede reflejar a veces la ausencia de esperanza y el temor al desengaño ${ }^{19}$. El postergado se reconcilia con las desigualdades sociales ajustando los deseos a las posibilidades. La métrica de los deseos, entonces, no posee mucha equidad.

c) En el caso de la elección Sen hace notar que ella es un reflejo del deseo, por lo que se le pueden aplicar las reflexiones del párrafo anterior. Por otra parte, la elección responde a motivaciones complejas y no refleja sólo consideraciones de bienestar ${ }^{20}$.

El denominador común de la crítica de Sen a la utilidad como criterio para determinar el nivel de vida es que ella -en sus tres versiones- tiene fuertes componentes subjetivos que pueden distorsionar el cálculo. Por cierto que esta imputación es inoponible a la opulencia, que se mide por bases objetivas. Explícitamente ${ }^{21}$ Sen reclama un criterio objetivo para medir el bienestar; la opulencia cumple con el requisito de la objetividad. Es cierto que puede argumentarse $\mathrm{e}^{22}$ que una persona puede ser opulenta y carecer de bienestar, dado lo variable de las características personales. Acepto que pueda decirse esto, pero si bien esa persona carece de bienestar no deja por ello de tener un buen nivel de vida. La variabilidad de las condiciones personales puede también hacer posible que una persona tenga bienestar sin tener un buen nivel de vida, como lo muestra, por ejemplo:

El caso de la madre Teresa: parece indiscutible que la madre Teresa de Calcuta no disfruta de un buen nivel de vida. En condiciones materiales muy desfavorables, y recargada de trabajo, dedica todos sus esfuerzos a ayudar a los pobres. A juzgar por sus declaraciones, sin embargo, ella disfruta de un alto grado de bienestar y se considera una mujer feliz.

Este ejemplo -y su opuesto, el del individuo opulento que carece de bienestarmuestra algo obvio: que el bienestar y el nivel de vida son dos cosas distintas. Y esto, a su vez. nos va a

${ }^{19}$ Sen. cit., págs. 10-11.

${ }^{20}$ Sen, cit., págs. 12-13.

${ }^{21}$ Amartya Sen, «Well-Being, Agency and Freedom», The Journal of Philophy, vol. LXXXII núm. 4. pág. 196.

${ }^{22}$ Sen, «Well-Being...», cit., pág. 196. 
permitir apreciar más adelante uno de los motivos por los que el modelo de Sen funciona deficientemente: el modelo fue concebido para dar cuenta del bienestar y extendido luego al nivel de vida, como si ambos fueran la misma cosa.

Pero veamos primero el modelo en sí. Sen evalúa el nivel de vida sobre la base de dos elementos, que denomina «funciones» y «capacidades». Las diversas condiciones de vida que una persona puede o no alcanzar se denominan funciones ${ }^{23}$. Las funciones pueden ser actividades (comer, leer), o estados de existencia (estar bien nutrido o libre de la malaria). El conjunto de funciones que una persona realmente alcanza se denomina el vector de función $^{24}$. La aptitud para alcanzar las funciones se denomina capacidad ${ }^{25}$. El conjunto de capacidades es el conjunto de vectores de función que está dentro del alcance de esa persona $^{26}$. La función es un logro, y la capacidad es una aptitud para alcanzar ese logro ${ }^{27}$. Las funciones están directamente relacionadas con las condiciones de vida, puesto que ellas son aspectos de las condiciones de vida. Para evaluar el nivel de vida parecería que hay que concentrarse entonces en las funciones y no en las capacidades, que son nociones de libertad, oportunidades reales. Pero Sen cree que las capacidades también desempeñan un papel directo, puesto que la idea del nivel de vida tiene un aspecto que no es independiente de la perspectiva de la libertad ${ }^{28}$. El modelo de Sen, pues, mide el nivel de vida sobre la base de las capacidades y funciones de los individuos.

Parecería que el argumento se desarrolla de esta forma:

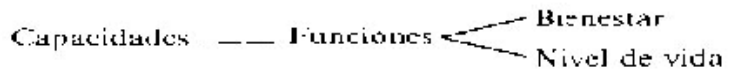

Aunque si tomamos en cuenta la cronología con que el argumento fue expuesto, el desarrollo es éste:

Capacidades — Funciones — Bienestar — Nivel de vida

${ }^{23}$ Sen, The Standard.... pág. 16.

${ }^{24}$ Sen, «Well-Being...», cit., págs. 196-198.

${ }^{25}$ Sen, The Standard..., cit. pág. 16.

${ }^{26}$ Sen, «Well-Being...», cit., pág. 201.

${ }^{27}$ Sen, The Standard..., cit. pág. 36.

${ }^{28}$ Sen, The Standard..., cit. pág. 36. 
Antes de abandonar el modelo -para comenzar a criticarlo en lugar de explicarloveamos un punto más acerca de las capacidades. Una de las capacidades que Sen menciona expresamente -y que toma de Adam Smith- es la de «aparecer en público sin avergonzarse ${ }^{29}$. Coincide con Adam Smith en que esto no depende de la opulencia, sino que los requerimientos de esta capacidad respecto de los bienes y de la riqueza dependen de la naturaleza de la sociedad en la que uno vive. Volveré sobre este ejemplo algo más adelante.

\section{La crítica al modelo de Sen}

El problema que enfrenta el modelo de Sen es el de la relación que existe entre la opulencia, por una parte, y las capacidades y funciones, por la otra. Sen acepta ${ }^{30}$ que la opulencia contribuye a las funciones, pero la relación es en realidad más estrecha. En rigor, en todo lo que se refiere al nivel de vida la opulencia determina las capacidades de un individuo. Y dada la relación entre las capacidades y las funciones, el nivel de vida puede remitirse directamente a la opulencia. Para apreciar este punto examinaré los casos principales en los que Sen se basa para establecer la pretendida diferencia entre las capacidades y la opulencia.

El primer caso es el de la salud. $\operatorname{Sen}^{31}$ se refiere al ejemplo del discapacitado que -con el mismo nivel de opulencia- no puede hacer muchas de las cosas que están al alcance de una persona normal. Lo que no se comprende, ante todo, es la vinculación entre este ejemplo y el nivel de vida. Sería extraño decir que un millonario discapacitado tiene un bajo nivel de vida; mucho más natural sería decir que no tiene -en todo- buena suerte. Volveré luego sobre este tema porque -como ya dije antes- creo que uno de los errores de Sen es extrapolar características del bienestar al nivel de vida. Es posible que el millonario discapacitado no tenga un alto nivel de bienestar, pero sí tiene un alto nivel de vida. Por otra parte, me pregunto si esta preocupación de Sen por el bienestar lo lleva -en definitiva- a buscar una suerte de «equiparación del bienestar». Si esto es así su proyecto es pasible de todas las críticas que Dworkin dirigió contra esta alternativa ${ }^{32}$.

\footnotetext{
${ }^{29}$ Sen, The Standard.... cit. pág. 17.

${ }^{30}$ Sen, «Well-Being...», cit., pág. 199.

${ }^{31}$ Sen, «Well-Being...», cit., pág. 196.

${ }^{32}$ Ronald Dworkin, «What is Equality?», Part 1. Philosophic and Public Affairs, vol. 10, núm. 3.
} 
El nivel de vida tiene importancia para la filosofía política porque se supone que una de las tareas posibles para un gobierno es la de intervenir para elevarlo, o para distribuirlo de un modo más igualitario. Por eso hay que distinguir dos tipos distintos de mala salud: $1 .^{\circ}$ ) aquella que es solucionable con dinero, como el caso de un individuo que requiere una operación cardíaca, perfectamente practicable si se pagan los honorarios del cirujano y del hospital;.$^{\circ}$ ) aquella que no es solucionable con dinero, como ciertos trastornos genéticos o la enfermedad que aqueja a nuestro infortunado millonario. El primer caso cuenta para determinar el bienestar, pero no el nivel de vida. Cuando la salud cuenta para el nivel de vida, entonces, es reducible a términos de opulencia. Cuando no lo es, no cuenta.

Sen nos pide que consideremos el caso de dos individuos (llamémoslos $x$ e $y$ ): $x$ tiene más ingresos que $y$, por lo que compra -y consume- más comida que éste. Pero su metabolismo es tal que, pese al consumo de comida, $x$ está más débil y desnutrido que $y$. Sen piensa que no puede decirse que $x$ tiene un mejor nivel de vida que $y^{33}$, y esto lo lleva a señalar ${ }^{34}$ que las variaciones interpersonales en «transformar» bienes en funciones son extremadamente comunes. El ejemplo me parece un caso claro de confusión entre el bienestar y el nivel de vida. Si el metabolismo de $x$ puede ser corregido médicamente, o si $x$ puede nutrirse adecuadamente comiendo el doble que $y$, entonces su situación se vincula al nivel de vida, pero esta vinculación deriva sólo del hecho obvio de que su caso se vincula con la opulencia. Si su metabolismo es irremediable, el único problema que resta es el de la cantidad de comida que debe comprar para nutrirse como $y$; y esto -repito- se relaciona con el nivel de vida sólo a través de la opulencia. Supongamos que y tenga una virtud metabólica, en lugar de un defecto metabólico como el que afecta a $y$. En el caso de $y$ prácticamente él no requiere comida para estar bien nutrido. De acuerdo al criterio de Sen, aunque $y$, apenas tenga el dinero suficiente para comprar su escasa cantidad de comida deberíamos decir que su nivel de vida es bueno, puesto que está bien nutrido. ¿Y qué diríamos del ejemplo que sigue?

El caso del avaro desnutrido: $x$ tiene enormes cantidades de dinero, pero ha concebido una pasión desordenada por él; sólo piensa en acumular cantidades aún mayores. Para lograrlo prescinde incluso de la alimentación adecuada y se encuentra en avanzado estado de desnutrición.

\footnotetext{
${ }^{33}$ Sen, The Standard..., cit. págs. 15-16.
}

${ }^{34}$ Sen. «Well-Being...», cit., pág. 199. 
¿Querría alguien decir que el nivel de vida del avaro es bajo? La respuesta es negativa y la explicación es sencilla: su nivel de vida está determinado (fundamentalmente) por su grado de opulencia.

El segundo caso en el que Sen se apoya para establecer las diferencias entre las capacidades y la opulencia es el de aparecer en público sin avergonzarse. Creo que tampoco esta circunstancia tiene nada que ver con el nivel de vida, como lo muestran los dos ejemplos que siguen:

El caso del londinense avergonzado: $x$ vive en Londres y disfruta de ingresos que le permiten llevar una vida acomodada. No puede cometer excesos, sin embargo, y su guardarropa no es ilimitado. Al recibir una invitación para una función de gala en la ópera $x$ debe rechazarla, puesto que le avergüenza presentarse en el teatro sin vestir frac.

El caso del indígena satisfecho: $y$ vive en una tribu amazónica sólo subsiste -magramentede la caza y de la pesca. La tribu requiere como único guardarropa un taparrabos, por lo que $y$ jamás debe rechazar una invitación por vergüenza.

¿Querría alguien decir que el nivel de vida de $y$ es más alto que el de $x$ ? En ese caso nos encontraríamos frente a esta paradoja: si todos los miembros de la sociedad son muy pobres, todos consumen lo mismo (muy poco) y, nadie tiene vergüenza. Consecuentemente, el nivel de vida de esa sociedad... ¡es alto! Pero si algunos miembros de la sociedad son muy ricos y otros no lo son tanto, los últimos pueden sentir vergüenza en alguna circunstancia. (-¡Cómo voy a estacionar mi Morris junto a su Rolls!»). Consecuentemente, el nivel de vida de esta sociedad... ¡es más bajo que el de la anterior! Pero en ese caso llegaríamos a la extraña conclusión de que sólo las sociedades absolutamente igualitarias tienen un nivel de vida alto, cualesquiera sea su nivel de ingreso. (Y concluiríamos que la igualdad no es sólo condición necesaria, sino también suficiente de un nivel de vida alto.)

Me parece que esto basta para refutar la idea de Sen, aunque todavía se puede agregar una cosa más: Sen critica al utilitarismo por emplear criterios subjetivos de evaluación y pretende basarse en criterios objetivos. Sin embargo, la sensación de vergüenza es subjetiva. El londinense $x$ puede avergonzarse de ir al teatro sin frac, mientras al londinense $z$ no le importa aparecer en el mismo acto sin corbata.

Nótese que no estoy diciendo que la capacidad de aparecer en público sin avergonzarse sea irrelevante para un individuo. $\mathrm{Si}$ 
$x$ es dueño de un solo traje y no tiene posibilidad de comprarse otro, sería beneficioso para él que la sociedad en la que vive viera con aprobación un guardarropa tan austero. El indígena satisfecho puede estar genuinamente satisfecho. Pero lo que objeto en Sen son dos cosas: $1^{\circ}$ ) que asigne una gran importancia a la capacidad en cuestión, y fundamentalmente, $2^{\circ} .^{)}$que la vincule con el nivel de vida. Veamos un ejemplo conectado con el del indígena:

El caso de Cranford: Elizabeth Gaskell nos ha descrito la vida en un ficticio pueblo rural de la Inglaterra del siglo XIX, Cranford. Nadie llevaba sus vestidos a la moda, fingían no advertir los signos de pobreza, y se consideraba vulgar el dar agasajos costosos.

Por cierto que un individuo pobre podía estar tan satisfecho en Cranford como nuestro indígena amazónico. Pero no por tener un nivel de vida elevado, sino por tener un grado de bienestar elevado, derivado -tal vez- de la ausencia de envidia. Imaginemos que un Gobierno convenza a sus súbditos de que se comporten como los habitantes de Cranford. El total de los bienes permanece invariable (no aumenta el grado de opulencia) y tampoco varía el tiempo libre. Los ciudadanos, sin embargo, están mucho más contentos que antes, porque ahora piensan que ésta es la forma adecuada de vivir. Yo no pretendo decir que la actitud de ese gobierno es incorrecta, pero ¿quién querría sostener que ha elevado el nivel de vida de sus súbditos? (Este caso es similar al del esclavo satisfecho que preocupó, entre otros, a Isaiah Berlin.)

Como dije antes, el problema de Sen radica en querer resolver dos problemas diferentes con los mismos conceptos. Primero identificó el bienestar personal de un individuo con el vector de función que obtiene ${ }^{35}$. Hasta aquí todo marchaba bien. Pero luego quiso emplear otra vez los conceptos de «funciones» y «capacidades» para resolver el problema -muy distinto por cierto- del nivel de vida. Tal vez Sen ha mostrado la verdad del refrán «El dinero no hace la felicidad», pero ciertamente no ha probado que «El dinero no hace el nivel de vida».

Ya expliqué cómo vinculaba Sen los conceptos de «funciones», «capacidades», «bienestar» y «nivel de vida». Voy a explicar ahora como vinculo yo esos conceptos, agregando el de «opulencia».

\footnotetext{
${ }^{35}$ Sen, «Well-Being...», cit., pág. 198.
} 


\section{Cuadro 2}

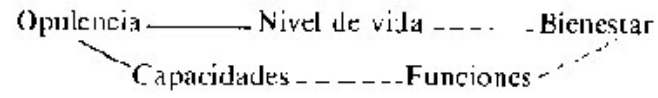

La opulencia influye, por una parte, en el nivel de vida, y lo hace de forma decisiva (de ahí la línea llena). Por otra parte, el nivel de vida puede influir en el bienestar, aunque no tan decisivamente (de ahí la línea punteada). A su vez, la opulencia influye decisivamente en las capacidades y las capacidades en las funciones (de ahí las dos líneas llenas). Las funciones, a su vez, influyen -aunque no tan decisivamente- en el bienestar (de ahí la línea punteada).

Si por mí fuera me quedaría solamente con el renglón superior del cuadro 2. Introduje el renglón inferior nada más que para vincular mi modelo con el de Sen y para mostrar otra cosa que puede tener importancia: que las capacidades y las funciones se vinculan, por un lado, con la opulencia y, por el otro, con el bienestar, pero no tienen nada que ver con el nivel de vida.

\section{Otros modelos posibles}

Hasta ahora el modelo de nivel de vida construido sobre la base de la opulencia y del tiempo libre parece operar mejor que el modelo de Sen, construido sobre la base de las capacidades y de las funciones. Pero esta prueba no es una garantía absoluta de corrección. ¿No hay alguna otra manera posible de considerar al nivel de vida? Recordemos que cualquier modelo debe respetar la condición de ser objetivo, en el sentido de que el nivel de vida no dependa de las consideraciones subjetivas de los individuos. Sen criticó al utilitarismo precisamente por este aspecto y Dworkin mostró con claridad las dificultades de la igualdad basada en el bienestar, dificultades que se reproducen si se pretende fundar el nivel de vida en el bienestar (especialmente si hay una preocupación igualitaria).

Pero ¿por qué no emplear el concepto de «necesidad» para caracterizar al nivel de vida? Cuanto más necesidades sean satisfechas, más alto se considera el nivel de vida. Griffin pretendió vincular el bienestar -en un sentido objetivo- con la satisfacción de las necesidades básicas, en la medida en que ellas retengan su importancia ${ }^{36}$. Ahora bien: ¿cuáles son las necesidades

\footnotetext{
${ }^{36}$ James Griffin, Well-Being, Oxford, Clarendon Press, 1986, págs. 42 y 52.
} 
básicas? Griffin ${ }^{37}$ expone que pueden ser los medios para cualesquiera sean los fines que el individuo tiene. Si esto fuera así, lo único objetivo es mensurar en dinero esas necesidades, lo cual vuelve a conducirnos al modelo de la opulencia. Tomemos la lista de necesidades de Braybrooke ${ }^{38}$ : algunas, como la comida y el descanso, se vinculan directamente con la opulencia y el tiempo libre. Otras, como las de excretar y tener actividad sexual, difícilmente puedan vincularse con el nivel de vida.

En realidad la idea misma de «necesidades» no está exenta de problemas. Las necesidades -lo acepto- parecen ser objetivas. Si $x$ necesita agua para sobrevivir, lo necesita lo desee o no lo desee, lo quiera o no lo quiera, lo sepa o no lo sepa. Las preferencias -en cambio- parecen ser subjetivas. Si $x$ prefiere la carne de vaca al pescado esto indica que desea más aquella que ésta, que quiere más aquélla que ésta, que sabe que le gusta más aquélla que ésta. Puesto que estamos buscando criterios objetivos para describir el nivel de vida, las necesidades parecen ser un candidato mejor que las preferencias. (Que las preferencias sean subjetivas no implica -por supuesto- que no haya criterios objetivos para determinarlas. Estoy pensando, por ejemplo, en el criterio de las preferencias reveladas.)

Pero si encaramos el tema desde el punto de vista de la política social la cuestión es más compleja. Satisfacer preferencias es una política no-paternalista. Satisfacer necesidades puede ser una política paternalista, si los individuos satisfechos no deseaban serlo. Un Testigo de Jehová puede necesitar una transfusión de sangre para sobrevivir, pero sin duda prefiere no recibirla.

Por otra parte, conceder prioridad irrestricta a las necesidades sobre las preferencias puede conducir a resultados perplejantes. De acuerdo a Braybrooke, como hemos visto, una de las necesidades es la de tener actividad sexual. Imaginemos una sociedad de tres individuos, $x, y$ y $z$. El individuo $x$ necesita actividad sexual, pero $y$ y $z$ prefieren no tener relaciones sexuales con $x$. Si las necesidades priman siempre frente a las preferencias habría que obligar a $y$ o a $z$ a satisfacer la necesidad de $x$, dejando de lado su preferencia en contrario.

De modo que el carácter objetivo de las necesidades no cuenta como una ventaja decisiva frente al carácter subjetivo de las preferencias. El modelo que he propuesto combina de un modo adecuado el objetivismo con el subjetivismo: $1 .^{\circ}$ ) $\mathrm{Al}$ medir el

\footnotetext{
${ }^{37}$ Griffin, cit., pág. 43.

${ }^{38}$ David Braybrooke. Meeting Needs, New Jersey, Princeton University Press, 1987, pág. 36.
} 
nivel de vida sobre la base de la opulencia presta atención al factor objetivo, puesto que el ingreso y la riqueza no dependen de apreciaciones subjetivas, como tampoco depende de ellas el cálculo de la cantidad de tiempo libre, que es el otro factor que mide el nivel de vida. (Lo que depende de apreciaciones subjetivas es el valor del tiempo libre.) 2..$^{\circ}$ ) Un sistema deseable permite que los individuos destinen su opulencia y su tiempo libre a la satisfacción de sus preferencias, lo que presta atención al factor subjetivo.

La caracterización del nivel de vida que he propuesto parece superior a las dos concepciones rivales que he examinado. Resta. sin embargo, un problema secundario, para el que preferí reservar el apartado siguiente.

\section{La libertad y las preferencias}

Cuando caractericé al nivel de vida lo hice en base a dos elementos: opulencia y tiempo libre. Completé luego el esquema introduciendo otros dos elementos que, aunque ajenos al nivel de vida, determinaban el grado de deseabilidad del sistema: la libertad de elección y la calidad de vida. Con la calidad de vida no parece que se presente problema alguno, pero no ocurre lo mismo con la libertad. Lo califiqué más arriba de problema «secundario en el sentido de que no afecta al concepto central que he tratado de determinar, que es el concepto de nivel de vida.

El problema reside en que cuando se aumenta en un sentido la libertad de elección puede disminuirse en otro sentido -a veces difícil de percibir- esa misma libertad. Entre imponer un culto religioso y permitir la libertad de cultos parece haber una gran diferencia, y pocos dudarían que la segunda alternativa incrementa la libertad de elección. Es cierto, por supuesto. Pero también la segunda alternativa disminuye en cierto sentido la libertad de elección, como lo muestra:

El caso del mártir frustrado: $x$ desea ser un mártir y morir por su religión. Pero no puede hacerlo, porque la sociedad $\mathrm{M}$ en que vive permite la libertad de culto, y nadie persigue a $x$ por practicar públicamente su religión.

En este sentido, es posible que $x$ prefiera vivir en la sociedad $\mathrm{N}$, que impone un culto oficial distinto al que $x$ practica y amenaza con el martirio al que no lo sigue.

Creo que este problema muestra lo siguiente: de los cuatro elementos que hemos estado considerando, tres de ellos pueden 
medirse objetivamente, a saber, la opulencia, el tiempo libre y la calidad de vida. Pero no ocurre lo mismo con la libertad de elección. No es sólo la cantidad de libertad lo que incide en la deseabilidad del sistema para un individuo determinado, sino el tipo de libertades permitidas.

\section{Final}

En conclusión: el nivel de vida se determina por la opulencia y el tiempo libre, mientras que la deseabilidad del sistema se determina por la libertad de elección y por la calidad de vida. (Por supuesto que -como señalé en el apartado 5- cuando digo que la deseabilidad del sistema se limita a la libertad de elección y a la calidad de vida estoy diciendo que esto es así cuando se comparan dos sistemas con idénticos niveles de vida. Si el nivel de vida varía, él mismo se convierte en un elemento a tener en cuenta para establecer la deseabilidad del sistema.) A la filosofía política le interesan los cuatro elementos, y no sólo los dos primeros. La tarea de un gobierno no concluye cuando ha logrado un nivel de vida aceptable; le resta preocuparse todavía por el grado de deseabilidad del sistema. En defensa del modelo propuesto puede decirse que es simple en su concepción y que parece funcionar mejor que los dos modelos alternativos que he examinado. ¿En qué sentido el modelo funciona mejor? Primero, me parece que reproduce de un modo más adecuado el significado usual de «nivel de vida». Si esta fuera su única virtud, sin embargo, el modelo sólo equivaldría a una correcta definición lexicográfica (aunque esto no es desdeñable). Pero no creo que sea todo. Porque, segundo, el modelo permite aclarar las ideas al distinguir entre el nivel de vida -adecuadamente definido- y la deseabilidad del sistema. De este modo es útil para orientar la acción del gobierno en beneficio de sus ciudadanos, separando las distintas tareas que se esperan de aquél y facilitando su labor ${ }^{39}$.

${ }^{39}$ Agradezco las observaciones de Guido Pincione, Horacio Spector y Hugo Zuleta. 